\title{
«De sens rassis» Essays in Honor of Rupert T. Pickens. Edited by Keith Busby, Bernard Guidot, and Logan E. Whalen
}

Maria Colombo Timelli

\section{(2) OpenEdition}

Journals

Édition électronique

URL : http://journals.openedition.org/studifrancesi/29908

DOI : $10.4000 /$ studifrancesi.29908

ISSN : 2421-5856

Éditeur

Rosenberg \& Sellier

Édition imprimée

Date de publication : 1 avril 2006

Pagination : 123-124

ISSN : 0039-2944

\section{Référence électronique}

Maria Colombo Timelli, « «De sens rassis» Essays in Honor of Rupert T. Pickens. Edited by Keith Busby, Bernard Guidot, and Logan E. Whalen », Studi Francesi [En ligne], 148 (XLX | I) | 2006, mis en ligne le 30 novembre 2015, consulté le 18 avril 2021. URL : http://journals.openedition.org/studifrancesi/29908 ; DOI : https://doi.org/10.4000/studifrancesi.29908 


\title{
«De sens rassis» Essays in Honor of Rupert T. Pickens. Edited by Keith Busby, Bernard Guidot, and Logan E. Whalen
}

\author{
Maria Colombo Timelli
}

\section{RÉFÉRENCE}

«De sens rassis» Essays in Honor of Rupert T. Pickens. Edited by KEITH BUSBY, BERNARD GUIDOT, and LOGAN E. WHALEN, Amsterdam-New York, Rodopi («Faux titre», 259), 2005, 753 pp.

1 Ce volume réunit une cinquantaine de contributions centrées pour la plupart sur la littérature française médiévale. Son ampleur même nous empêche d'en rendre compte dans les détails: nous nous limiterons donc - tout en le regrettant - à donner un inventaire des auteurs et des sujets concernés.

La matière arthurienne se taille la part du lion, avec de très nombreuses études:

3 GERARD J. BRAULt (The Prose 'Lancelot' and the 'Galehot Roll of Arms', pp. 17-34; il s'agit de l'armorial arthurien du ms. Paris, BnF fr. 32753: Les armes du Roy Galehot et de trente roys qu'il conquesta).

CARLETON W. CARRoll (One Text, Two Scribes: Manuscript P of 'Erec et Enide' (Paris, BnF, fr. 375, pp. 109-124: étude des pratiques graphiques - forme de certaines lettres, abréviations, ponctuation, graphies - du deuxième scribe du ms. $P$ ).

5 CAROL J. CHASE (Christ, the Hermit and the Book: Text and Figuration in the Prologue to the 'Estoire del Saint Graal', pp. 125-147: analyse du contenu des enluminures qui illustrent le prologue de l'Estoire dans les manuscrits de la BnF et de la Bibliothèque de l'Arsenal). 

Perceforest, situés dans la première partie du livre II, seraient des récritures directes de Chrétien de Troyes, l'une du Conte du Graal, l'autre du Chevalier au Lion). en prose du Chevalier de la Charrette transmise par trois mss. du XIV ${ }^{\mathrm{e}}$ siècle du LancelotGraal: Arsenal 3480, BnF fr. 119, BnF fr. 122).

DOUGLAS Kelly (How Did Guiolete Come to Court? Or, the Sometimes Inscrutable Paths of Tradition, pp. 309-323: réflexions sur certaines notions problématiques: réception des œuvres médiévales, genres littéraires, modèles prétendus ou réels). AURÉLIE KOSTKA (La ville, un Autre Monde? Discontinuité de l'espace urbain dans les romans arthuriens, pp. 353-363: exemples de 'cités' tirés essentiellement de la Première Continuation de Perceval et du Perlesvaus). NORRIS J. LACY (On Armor and Identity: Chrétien and Beyond, pp. 365-374: réflexion sur le rapport entre armes / armures et identité des chevaliers arthuriens; le corpus comprend les romans de Chrétien, le Chevalier as deux espees, les Merveilles de Rigomer, le Tristan en prose).

BARBARA N. SARGENT-BAUR (Rewriting 'Cligés', pp. 577-588: échos du Tristan de Thomas dans Cligés et de Cligés et Tristan dans Jehan et Blonde de Philippe de Remi). pp. 667-681: Galaad entretient une double 'ressemblance' - clé fondamentale de lecture de la Queste - avec Lancelot, son père, et avec le Christ).

LORI J. WALTERS (The King's Example: Arthur, Gauvain, and Lancelot in 'Rigomer' and Chantilly, Musée Condé 472 (anc. 626), pp. 699-717: ce manuscrit exceptionnel, qui réunit onze romans arthuriens, en vers et en prose, constitue un 'cycle de Gauvain', où les rois, et Gauvain par-dessus tous, offrent un modèle à imiter fondé essentiellement sur la parole).

LENORA D. WOLFGANG (The Manuscripts of the 'Chevalier de la Charrette (Lancelot)' of Chrétien de Troyes. Preliminary remarks to a new edition: The case of ms. E. Part II, pp. 729-753; discussion des lacunae du ms. E; la première partie a paru dans les Mélanges Philippe Ménard, Paris, Champion, 1998, pp. 1477-88). souligne l'importance de l'idée d'unité dans l'organisation narrative de l'œuvre de Béroul, au-delà de la pluralité de ses 'modèles'; voir aussi l'article du même D.M. dans Medioevo Romanzo, 25, 2002).

19 Et sur Marie de France:

20 JUNE HALL MCCASH (Philomena's Window: Issues of Intertextuality and Influence in Works of Marie de France and Chrétien de Troyes, pp. 415-430: relève des différences considérables 
dans le traitement du mythe ovidien dans Philomela, attribué à Chrétien, et Laüstic de Marie de France).

EMANUEL J. MICKEL (Marie's Use of Monologue and Dialogue in the 'Lais', pp. 467-489: malgré leur brièveté, les Lais sont riches en monologues et surtout en dialogues, qui permettent à Marie de dramatiser l'histoire).

LOGAN E. WHALEN (Marie de France and the Ancients, pp. 719-728: l'analyse des prologues de Marie semble montrer une préférence pour les techniques rhétoriques de la memoria et de la descriptio).

Font le lien entre la matière arthurienne et la matière épique:

ALAIN CORBELLARI (Les jeux de l'anneau: fonctions et trajets d'un objet emblématique de la littérature narrative médiévale, pp. 157-167: la présence et la fonction de l'anneau sont rapidement analysées dans les textes tristaniens, la Chanson de Roland - version de Paris, la Chanson d'Aspremont).

JACQUES E. MERCERON (Le miracle et les gués de l'aubépine: signe de salut et seuils de l'aventure dans la matière de France et de Bretagne, pp. 445-465: l'aubépine revêt deux significations fondamentalement différentes dans la matière carolingienne, où elle est un signe de la générosité de Dieu envers ses fidèles, et dans la matière de Bretagne, où elle marque l'accès vers l'Autre Monde et l'initiation chevaleresque).

Conformément aux centres d'intérêt du destinataire de ce volume, d'autres études concernent spécialement l'épopée:

ROBERT FRANCIS COOK (Notes sur le texte du 'Bâtard de Bouillon': le rôti et les fleurs; le nom de Bohémond, pp. 149-156: il s'agit de deux propositions de corrections, respectivement au v. $5266 \mathrm{du}$ ms. BnF fr. 12552 et à la graphie du nom Bujemons/t, à lire désormais Buiemons/t).

BERNARD GUIDOT (La famille de Narbonne dans 'Elie de Saint Gilles', pp. 233-247: présents dans la geste d'Elie de Saint Gilles, certains membres de la famille de Narbonne n'y jouent cependant pas toujours le rôle de prestige qui leur est normalement attribué).

EDWARD A. HEINEMANN (More on Speech Presentation in the 'Charroi de Nîmes': In Which Otran and Harpin Begin to Speak, pp. 249-264: analyse des vers où les verbes du dire introduisent les mots d'Otran et d'Harpin, laisses XLII-LII du Charroi de Nîmes).

CATHERINe M. Jones (Polyglots in the 'chansons de geste', pp. 297-307: étudie le motif de la polyglossie dans: Aiol, Aliscans, Guillaume, Entrée d'Espagne, Gaufrey, Girart de Roussillon, Guy de Bourgogne, Prise d'Orange).

31 WILLIAM W. KIBLER ('Huon de Bordeaux' in its Manuscripts, pp. 325-337: étude comparative des deux versions, transmises par le ms. de Tours, suivi par Pierre Ruelle dans son édition de 1960, et le ms. de Paris, choisi par le même W. Kibler dans l'édition bilingue, en collaboration avec François Suard, Paris, Champion, 2003).

MURIEL OTT (Le siège de Narbonne dans le 'Siège de Barbastre' et 'Buevon de Conmarchis', pp. 491-508: analyse comparée de l'épisode du siège de Narbonne dans Barbastre et dans le remaniement d'Adenet le Roi).

MARY JANE SCHENCK (Spectacles of Violence: The Trials of Ganelon, pp. 589-603: lecture 'politique' du procès de Ganelon selon la version d'Oxford et le ms. de Châteauroux de la Chanson de Roland).

D’autres œuvres médiévales font l'objet des réflexions de: 


\section{9-182: réflexions
traduction anglaise)}

46 $\mathrm{du}$ XIII $^{\mathrm{e}}$ siècle). sur leur contenu). hagiographique). tome III, pp. 516-545).

Paul BRETEl (Moines et religieux dans les contes de la 'Vie des Pères', pp. 35-50: analyse des transformations introduites dans les trois parties de la Vie des Pères, reflet des changements dans les mentalités religieuses et les préoccupations de l'Eglise au cours

KEITH BUSBY (Filling in the Blanks: The Missing Miniatures in BnF, fr. 15101 of 'Florimont', pp. 85-95: analyse des emplacements réservés aux miniatures dans le ms., et hypothèses

LAURENCE MATHEY-MALle (L'étymologie dans le 'Roman de Rou' de Wace, pp. 403-414: présentation de quelques étymologies du Rou [Normant, Danois, Luna, 'mostier' Toz Sains ou Sainte Pais, Pié de Cers, surnom de Henri $1^{\text {er }}$ et signification de ce procédé à l'intérieur de l'écriture 'historique' de Wace).

PHILIPPE MÉNARD (Les 'Prophéties de Merlin' et l'Italie au XIII siècle, pp. 431-444: cette œuvre, composée sans doute entre 1272 et 1279 , contient des allusions aux événements passés de la Marche de Trévise; inscrite dans la littérature apocalyptique, son écriture est aussi marquée par l'hermétisme).

DUNCAN ROBERTSON (Seasons of Solitude: The Anglo-Norman Verse 'Life of St. Giles', pp. 557-565: discussion sur le 'genre' d'une Vie anglo-normande du XII ${ }^{\mathrm{e}}$ siècle, réfection amplifiée d'un texte latin du $\mathrm{X}^{\mathrm{e}}$, située à la convergence de la biographie 'sacrée' et du roman

SARA STURM-MADDOX ('Signeur, vous qui l'oevre savés': Amadas, Ydoine, and the Wiles of Women, pp. 605-616: analyse des comportements d'Ydoine, rendue 'subtile et sage' par amour).

Des revisitations de la littérature médiévale sont présentées par:

JEAN DUfOURnET (Gaston Paris et Villon. Entre Auguste Longnon et Marcel Schwob, pp. 183-199: réflexions sur la réception de François Villon par Gaston Paris et Auguste Longnon).

FRANÇOIS SUARD (Alexandre le Grand et Malraux, pp. 617-628: analyse d'un texte d'André Malraux sur le grand Macédonien, publié dans la «Pléiade», éds. Guyard et Trécourt,

On trouvera encore des réflexions philologiques ou des éditions de textes par:

PETER F. DEMBOWSKI (What is Critical in Critical Editions? The Case of Bilingual Editions, pp. TONY HUNT (Wordplay before the 'Rhétoriqueurs', pp. 283-296; avec édition d'un poème de Walther of Bibbesworth en l'honneur de la Vierge).

7 SAMUel N. ROSENBERG (French Songs in Occitan Chansonniers: Mahieu le Juif in ms. O (Rome, Biblioteca Apostolica Vaticana, vaticani latini 3208, pp. 567-575: édition de la chanson 'provençalisée' de Mahieu le Juif Per gran franchisa me conven chantar [Par grant franchise me convient chanter]). 